\title{
The role of maternal health care services as predictors of time to modern contraceptive use after childbirth in North West Ethiopia: Application of the shared frailty survival analysis --Manuscript Draft--
}

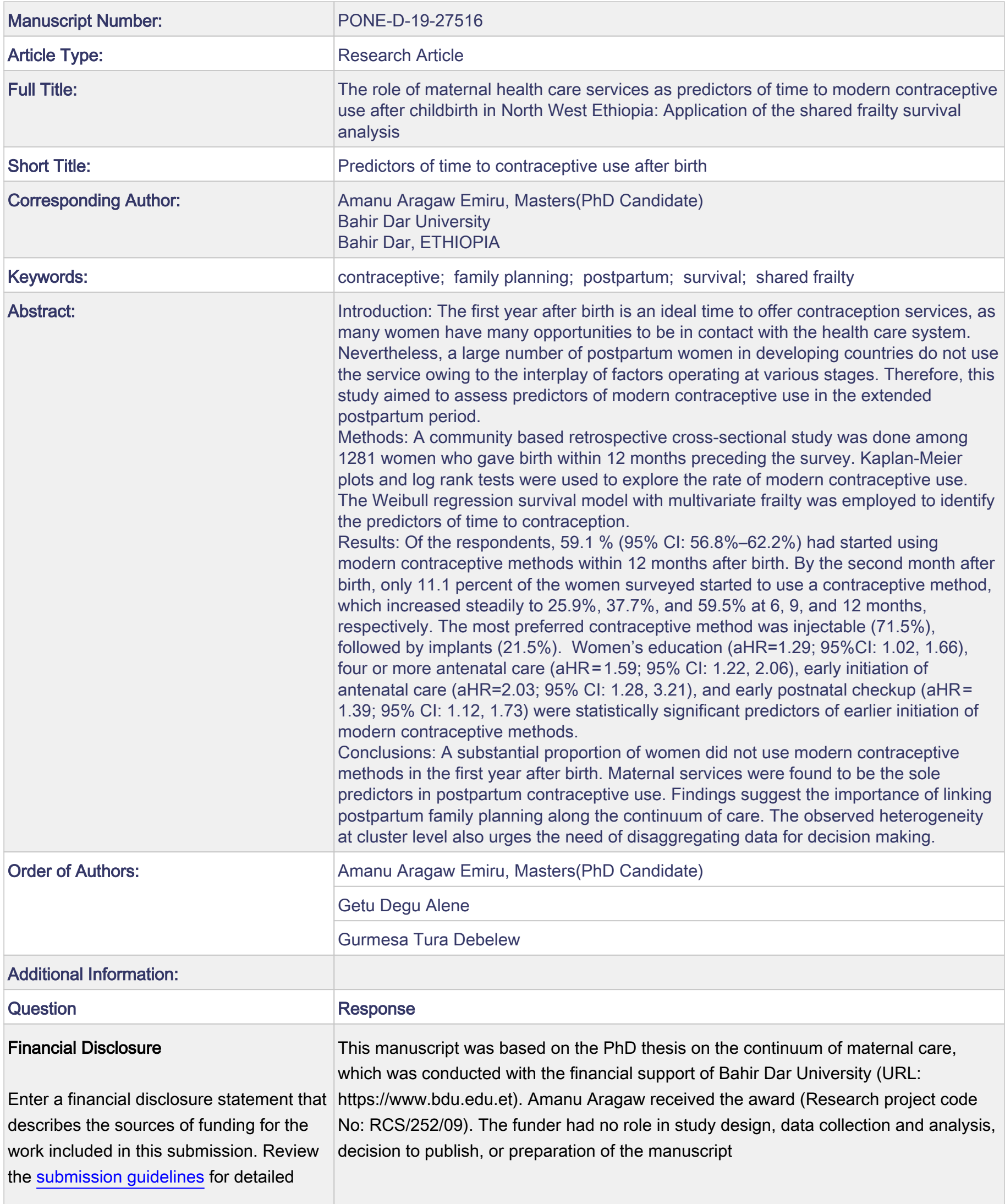


requirements. View published research articles from PLOS ONE for specific examples.

This statement is required for submission and will appear in the published article if the submission is accepted. Please make sure it is accurate.

\section{Unfunded studies}

Enter: The author(s) received no specific funding for this work.

\section{Funded studies}

Enter a statement with the following details:

- Initials of the authors who received each award

- Grant numbers awarded to each author

- The full name of each funder

- URL of each funder website

- Did the sponsors or funders play any role in the study design, data collection and analysis, decision to publish, or preparation of the manuscript?

- NO - Include this sentence at the end of your statement: The funders had no role in study design, data collection and analysis, decision to publish, or preparation of the manuscript.

- YES - Specify the role(s) played.

\section{* typeset}

\section{Competing Interests}

The authors have no competing interest

Use the instructions below to enter a competing interest statement for this submission. On behalf of all authors, disclose any competing interests that could be perceived to bias this work-acknowledging all financial support and any other relevant financial or nonfinancial competing interests.

This statement will appear in the published article if the submission is accepted. Please make sure it is accurate. View published research articles from PLOS ONE for specific examples. 


\section{NO authors have competing interests \\ Enter: The authors have declared that no competing interests exist. \\ Authors with competing interests \\ Enter competing interest details beginning with this statement: \\ I have read the journal's policy and the authors of this manuscript have the following competing interests: [insert competing interests here] \\ * typeset}

\section{Ethics Statement}

Enter an ethics statement for this submission. This statement is required if the study involved:

- Human participants

- Human specimens or tissue

- Vertebrate animals or cephalopods

- Vertebrate embryos or tissues

- Field research

Write "N/A" if the submission does not require an ethics statement.

General guidance is provided below.

Consult the submission guidelines for detailed instructions. Make sure that all information entered here is included in the Methods section of the manuscript.
This study was approved by the Research Ethical review committee of the College of Medicine and Health Sciences, Bahir Dar University (reference number: 088/18-04). Letters of permission were secured from the Amhara Regional State Health Bureau and respective health offices. Also, oral informed consent was received from each study participant. The data obtained from each study participants was kept confidential throughout the process of study, and the name of the participants was replaced by code. 


\section{Format for specific study types}

Human Subject Research (involving human participants and/or tissue)

- Give the name of the institutional review board or ethics committee that approved the study

- Include the approval number and/or a statement indicating approval of this research

- Indicate the form of consent obtained (written/oral) or the reason that consent was not obtained (e.g. the data were analyzed anonymously)

\section{Animal Research (involving vertebrate}

\section{animals, embryos or tissues)}

- Provide the name of the Institutional Animal Care and Use Committee (IACUC) or other relevant ethics board that reviewed the study protocol, and indicate whether they approved this research or granted a formal waiver of ethical approval

- Include an approval number if one was obtained

- If the study involved non-human primates, add additional details about animal welfare and steps taken to ameliorate suffering

- If anesthesia, euthanasia, or any kind of animal sacrifice is part of the study, include briefly which substances and/or methods were applied

\section{Field Research}

Include the following details if this study involves the collection of plant, animal, or other materials from a natural setting:

- Field permit number

- Name of the institution or relevant body that granted permission

\section{Data Availability}

Yes - all data are fully available without restriction

Authors are required to make all data underlying the findings described fully available, without restriction, and from the time of publication. PLOS allows rare exceptions to address legal and ethical concerns. See the PLOS Data Policy and FAQ for detailed information. 
A Data Availability Statement describing where the data can be found is required at submission. Your answers to this question constitute the Data Availability Statement and will be published in the article, if accepted.

Important: Stating 'data available on request from the author' is not sufficient. If your data are only available upon request, select 'No' for the first question and explain your exceptional situation in the text box.

Do the authors confirm that all data underlying the findings described in their manuscript are fully available without restriction?

Describe where the data may be found in All relevant data are within the manuscript and figures.

full sentences. If you are copying our sample text, replace any instances of $X X X$ with the appropriate details.

- If the data are held or will be held in a public repository, include URLs, accession numbers or DOls. If this information will only be available after acceptance, indicate this by ticking the box below. For example: $A$ II $X X X$ files are available from the $X X X$ database (accession number(s) $X X X, X X X$.).

- If the data are all contained within the manuscript and/or Supporting Information files, enter the following: All relevant data are within the manuscript and its Supporting Information files.

- If neither of these applies but you are able to provide details of access elsewhere, with or without limitations, please do so. For example:

Data cannot be shared publicly because of $[X X X]$. Data are available from the $X X X$ Institutional Data Access / Ethics Committee (contact via $X X X)$ for researchers who meet the criteria for access to confidential data.

The data underlying the results presented in the study are available from (include the name of the third party 
and contact information or URL).

- This text is appropriate if the data are owned by a third party and authors do not have permission to share the data.

* typeset

Additional data availability information: 
Predictors of time to contraceptive use after birth

1 The role of maternal health care services as predictors of time to 2 modern contraceptive use after childbirth in North West 3 Ethiopia: Application of the shared frailty survival analysis

4

5

19.

0

Amanu Aragaw Emiru, ${ }^{1 *}$ Getu Degu Alene ${ }^{2}$, Gurmesa Tura Debelew ${ }^{3}$

${ }^{1}$ Department of Reproductive Health and Population Studies, College of Medicine and Health sciences, Bahir Dar University, Bahir Dar, Ethiopia. Email: amanuaragaw @ yahoo.com

${ }^{2}$ Department of Epidemiology and Biostatistics, College of Medicine and Health sciences, Bahir Dar University, Bahir, Ethiopia. Email: adgetu123@yahoo.com

${ }^{3}$ Department of Population and Family Health, Faculty of Public Health, Institute of Health, Jimma University, Jimma, Ethiopia. Email: gurmesatura@ gmail.com

* amanuaragaw@yahoo.com . 
Predictors of time to contraceptive use after birth

\section{Abstract}

28 Introduction: The first year after birth is an ideal time to offer contraception services, as

29

30

31

32

many women have many opportunities to be in contact with the health care system. Nevertheless, a large number of postpartum women in developing countries do not use the service owing to the interplay of factors operating at various stages. Therefore, this study aimed to assess predictors of modern contraceptive use in the extended postpartum period.

Methods: A community based retrospective cross-sectional study was done among 1281 women who gave birth within 12 months preceding the survey. Kaplan-Meier plots and log rank tests were used to explore the rate of modern contraceptive use. The Weibull regression survival model with multivariate frailty was employed to identify the predictors of time to contraception.

Results: Of the respondents, $59.1 \%$ (95\% CI: 56.8\%-62.2\%) had started using modern contraceptive methods within 12 months after birth. By the second month after birth, only 11.1 percent of the women surveyed started to use a contraceptive method, which increased steadily to $25.9 \%, 37.7 \%$, and $59.5 \%$ at 6,9 , and 12 months, respectively. The most preferred contraceptive method was injectable (71.5\%), followed by implants $(21.5 \%)$. Women's education $(\mathrm{aHR}=1.29 ; 95 \% \mathrm{CI}: 1.02,1.66)$, four or more antenatal care $(\mathrm{aHR}=1.59 ; 95 \% \mathrm{CI}$ : $1.22,2.06)$, early initiation of antenatal care (aHR=2.03; 95\% CI: $1.28,3.21)$, and early postnatal checkup (aHR $=1.39 ; 95 \%$ CI: 1.12, 1.73) were statistically significant predictors of earlier initiation of modern contraceptive methods.

Conclusions: A substantial proportion of women did not use modern contraceptive methods in the first year after birth. Maternal services were found to be the sole predictors in postpartum 
48 contraceptive use. Findings suggest the importance of linking postpartum family planning along

49 the continuum of care. The observed heterogeneity at cluster level also urges the need of 50 disaggregating data for decision making.

51 Key words: contraceptive, family planning, postpartum, survival, shared frailty

52

53

54

55

56

57

58

59

60

61

62

63

64 


\section{Introduction}

66 At the turn of the $21^{\text {st }}$ century maternal mortality remains a major public health challenge for 67 many developing countries, and nowhere are global inequalities more starkly clear than in maternal death. The low-income countries accounted for $99 \%$ of these deaths of which two-third occurs in Sub-Saharan Africa where Ethiopia lies [1,2]. At 412 deaths per 100,000 live births[3], Ethiopia's maternal mortality ratio is one of the highest even by the standard of developing countries [4].

However, it is known that many of the maternal deaths can be prevented with appropriate maternal care during pregnancy, delivery and post natal periods $[5,6]$. With this in view, women access health services more often during the time of pregnancy, delivery, and the first year after birth than other periods [7].These points of contact, whether part of a routine or emergency care, are opportunities for providers to screen for, counsel, and hence to address the contraceptive needs of postpartum women and couples[8]. Therefore, promoting contraceptive use during each contact in the continuum is considered an important strategy for addressing the widespread unmet needs in family planning [9].

Despite this, the vast majority of postpartum women in developing countries missed the opportunities at each point of contact. With this, postpartum women are among those with the greatest unmet need for family planning than other periods $[6,10,11]$. In Ethiopia, unmet need for family planning in the postpartum period is also much higher than women outside of the extended postpartum period; while $16.2 \%$ women, in general, do have unmet need for family planning[12], the unmet need in the first year after childbirth reaches as high as 44 percent [13].

Women's decision to use modern contraceptives in the first year of postpartum is influenced by a complex array of factors within the community and health system[14,15]. Postpartum women 
88 often do not realize that they are at risk of pregnancy when they are amenorrheic or breastfeeding

89 [15]. Postpartum contraception adoption has also a social connotation; the postpartum period is

90 the time during which a woman needs to adjust herself to new roles to care for her newborn [16].

91 Furthermore, male dominance and subordination of women, as well as mistaken beliefs, and

92 religious faith of the population are other impediments to the acceptance of contraception in

93 developing countries, including Ethiopia [15].

94 Beyond the socio-cultural barriers, insufficient contraceptive method mixes, and poor family 95 planning service integration with other health services in many settings also appear to be 96 formidable obstacles remaining to be correct $[8,17]$.

97 Several studies have been carried out on postpartum contraceptive use in the study area in 98 particular and Ethiopia at large [18-20]; yet, most of the earlier studies, if any, have ignored the 99 hierarchical facts. It is, however, evidenced that clustering (frailty) has an effect on modeling the 100 predictors of time to contraception[21]. Consequently, modeling time to contraceptive use 101 ignoring the frailty terms may lead to biased estimates of parameters and their respective 102 standard errors.

103 Furthermore, according to the current Ethiopian Demographic and health survey (EDHS), 104 regional disparities in contraceptive adoption have been reported, with higher rates observed in 105 the Amhara region(where the study area is found) and Addis Ababa(the capital city of the 106 country) than the rest of the regions[3]. Nevertheless, this was a survey report rather than an 107 empirical study, and the contribution of explanatory factors was not examined.

108 In this regard, addressing these gaps would have significant implications for policymakers, 109 health planners, and clinicians. Therefore, considering the hierarchal nature of our data we 110 attempted to provide an understanding of factors associated with the timing of postpartum 111 contraceptive use using a shared frailty survival model. 
Predictors of time to contraceptive use after birth

\section{Materials and methods}

113 Study setting

114 The study was carried out in West Gojjam zone, which is one of the eleven zones found in the

115 Amhara region of Ethiopia. Administratively, the zone is subdivided into 13 rural districts and 2

116 town administrations with a projected total population of 2,611,925 $(2,194,017$ rural and 417,908

117 urban) people. The number of females in the reproductive age group was 615,892 , accounting for

$11823.58 \%$ of the total population[22].

119 The zone had over 598 health facilities (6 public primary hospitals, 103 health centers, 374

120 functional health posts, 114 private clinics, and 1 private hospital) at the time of the survey.

121 Family planning, antenatal care, labor and delivery, and postnatal services are provided free of

122 charge in all the public health facilities[22].

\section{Study design and population}

124 A community-based retrospective cross-sectional study was conducted on reproductive-aged 125 women whose most recent birth was within 12 months preceding the survey.

\section{Sample size and sampling procedure}

127 The required sample size was done through the STAT CALC program of the Epi-Info statistical 128 package V.7.0. This study was part of a large study done on the continuum of maternal health 129 care with multiple objectives. For each objective alternative sample sizes were computed 130 considering both the double and single population formulas; the detail of the sample size 131 calculation and sampling procedure is publicly posted in the research square. Of the alternative 132 sample sizes computed based on different indicators, the largest sample size (1294 women) was 
133 obtained when considering the following assumptions; 95\% confidence level, 4\% margin of error,

$13416.5 \%$ proportion of PNC utilization[23], design effect of 2 , and $10 \%$ non-response rate. 135 However, during the time of data collection 1337 women who met the inclusion criteria were 136 included in this study to increase the power of the study.

137 A multistage sampling technique was used to identify the study participants. First, five out of 138 fifteen districts in the Zone (four rural districts and one town administration) were selected using 139 simple random sampling. Second, thirteen kebeles (the smallest administrative units in Ethiopia) 140 were chosen randomly by taking in to account the number of kebeles in each district. Then, a 141 complete list of deliveries that took place within 12 months before the survey was identified 142 from the family folder of health extension workers in the respective kebeles. Finally, 1337 143 eligible women who met the inclusion criteria were selected.

\section{Study Variables and measurement}

145 The outcome variable was modern contraceptive use within 12 months following the last birth. A 146 woman who started using modern contraceptives was coded as " 1 ", and otherwise "0".

147 The explanatory variables included socioeconomic variables (such as place of residence, 148 maternal/paternal education, household wealth index, and Primary Health care (PHC) facilities 149 per 25,000 populations at district level), demographic characteristics (age of women, occupation 150 of women and husbands), and reproductive variables (such as desirability of the pregnancy, 151 family size, birth interval, number and timing of antenatal visits, mode of delivery, and Postnatal 152 care.

153 The wealth index was generated from the household's cumulative living standard based on 154 ownership of specified assets using factor analysis and was later categorized into terciles (poor, 155 middle and rich). 
Predictors of time to contraceptive use after birth

156 The two quantitative terms, survivor function $\mathrm{S}(\mathrm{t})$ and hazard function $\mathrm{h}(\mathrm{t})$, are important in any

157 survival analysis[21]. In relation to our study, the survivor function is the probability that a 158 postpartum woman "survives" longer than some specified time " $\mathrm{t}$ " without started taking modern 159 contraceptive methods after childbirth, while the hazard function gives the instantaneous 160 potential per unit time to start using modern contraception after time "t", given that the woman 161 had not started taking any modern contraceptive up to time " $\mathrm{t}$ ".

\section{Data collection process}

163 The household data were collected using a pre-tested interviewer-administered questionnaire, 164 developed in the local language, Amharic. Fifteen nurses and five public health officers were 165 deployed as data collectors and supervisors, respectively after receiving two days of intensive 166 training. Data regarding socio-economic, demographic, and reproductive characteristics were 167 collected among women who gave birth (either at home or in a health facility) to a baby within 16812 months before the survey. Besides, the number of PHC providing maternity and reproductive 169 health services per total population was assessed at the district level, and the result had been 170 linked to the individual woman in the corresponding household survey.

\section{Data processing and analysis}

172 The analysis was done using STATA 14.0. Both descriptive statistics and survival analysis 173 techniques were used in analyzing the data. First, an assessment of the time-to-modern 174 contraceptive use after birth was done using life tables based on the Kaplan-Meier (K-M) 175 estimate. Second, the Log-rank Chi-square test was used to examine the differences in the 176 survival curves for different categories of each study variable. Third, the multivariate (or shared 
177 frailty) survival analysis was done by assuming different parametric distributions for the baseline 178 hazard function and using gamma for frailty distributions.

179 The shared frailty approach is a conditional independence model for time to event data, where 180 the frailty term (the random effect) is common to all subjects in a cluster [21]. In our study 181 women who were living in the same cluster (kebele) were more likely to have outcomes (post182 partum contraceptive use) that are correlated with one another, and, thus independence between 183 event times cannot be assumed. Moreover, it is unlikely to include all the relevant covariates in 184 the model. With that in mind, using the cox proportional hazard model could not account for all 185 the variability in the observed failure times. Therefore, it was reasonable to apply the shared 186 frailty survival model, that accounts for heterogeneity caused by unmeasured covariates, as an 187 alternative to the standard cox survival model $[21,24]$. The conditional hazard function for the 188 Weibull shared frailty survival model used in this study is defined as:

$$
\lambda\left(\mathbf{t}_{\mathrm{ij}} / \mathbf{Z}_{\mathrm{i}}\right)=\mathbf{Z}_{\mathbf{i}} \lambda_{0}\left(\mathbf{t}_{\mathrm{ij}}\right) \exp \left(\boldsymbol{\beta}^{\prime} \mathbf{x}_{\mathbf{i j}}\right), \mathrm{i}=1 \ldots, \mathrm{n} ; \mathrm{j}=1, \ldots, \mathrm{k}_{\mathrm{i}}
$$

Where; $i$ indicate the $i^{\text {th }}$ cluster (kebele), $j$ indicates the $j^{\text {th }}$ individual in the $i^{\text {th }}$ cluster, $\mathrm{t}_{\mathrm{ij}}$ is the 191 observed failure time of a right censoring scheme for the $\mathrm{k}^{\text {th }}\left(\mathrm{k}=1, \ldots, \mathrm{n}_{\mathrm{j}}\right)$ observation in the $\mathrm{i}^{\text {th }}$ 192 cluster, $\lambda 0()=.\mathrm{pt}^{\mathrm{p}-1}$ is the baseline hazard, $\mathrm{X}_{\mathrm{ij}}$ is the vector of covariates for subject $j$ in cluster $i$, $193 \beta^{\prime}$ is a vector of regression coefficients, and 7: is the frailty term. In this study the frailty $\mathrm{Z}_{\mathrm{j}}$ was 194 supposed to follow a gamma distribution $\mathrm{g}(\mathrm{z} ; \theta, \theta)$, which is the most common and widely used 195 distribution for determining the frailty effect[24].

196 The Akaike Information Criterion (AIC) was used to select the appropriate model. Further, the 197 Cox-Snell residual plot analysis was done to evaluate the overall model fitness. Furthermore, 198 interaction between the independent variables for contraceptive use was tested. Finally, the 199 frailty effects, Kendall's Tau, and hazard ratio at 95\% confidence interval were estimated for the 200 selected model. 
Predictors of time to contraceptive use after birth

201

202

203

204

205

206

207 participants was replaced by code.

\section{Results}

\section{Background characteristics}

211 A total of 1281 reproductive- age women participated in this study giving a response rate of

$21295.8 \%$. More than half $674(52.6 \%)$ of the women were in the age group of $25-34$ years with a

213 mean $( \pm$ SD) age of $30.3( \pm 6.0)$ years. Little more than three-quarters, $978(76.3 \%)$, of the

214 sampled women were rural residents, and 862(67.3\%) of them belonged to the lower two wealth

215 quintiles.

216 Concerning the reproductive characteristics, 511(39.9\%) of them had at least 4 ANC visits,

217 194(15.1\%) had their first ANC visit within the first four months after conception. Also, the

218 highest proportion, 672(52.5\%) of women had deliveries at home and almost a similar proportion

219 of $719(56.1 \%)$ women did not have any health check within the six weeks of postpartum (Table $2201)$.

221 
Predictors of time to contraceptive use after birth

222 Table 1. Background characteristics of postpartum women in West Gojjam Zone,

223 Northwest Ethiopia, 2018 $(\mathbf{n}=1281)$.

\begin{tabular}{|c|c|c|c|}
\hline \multicolumn{4}{|c|}{ Survival Status } \\
\hline Variables & $\begin{array}{c}\text { Failures } \\
\text { (contraceptive } \\
\text { users) } \\
n=762\end{array}$ & $\begin{array}{l}\text { Censored } \\
\text { (Nonusers ) } \\
\mathbf{n}=\mathbf{5 1 9}\end{array}$ & Total \\
\hline \multicolumn{4}{|l|}{ Residence } \\
\hline Rural & 551 & 427 & $978(76.3)$ \\
\hline Urban & 211 & 92 & $303(23.7)$ \\
\hline \multicolumn{4}{|l|}{ Age of the women } \\
\hline $15-24$ years & 165 & 61 & $226(17.6)$ \\
\hline 25-34 years & 408 & 266 & $674(52.7)$ \\
\hline$>=35$ years & 189 & 192 & $381(29.7)$ \\
\hline \multicolumn{4}{|c|}{ Education status of women } \\
\hline No education & 409 & 415 & $824(64.3)$ \\
\hline Primary education & 221 & 85 & $306(23.9)$ \\
\hline Secondary and above & 132 & 19 & $151(11.8)$ \\
\hline \multicolumn{4}{|c|}{ Education status of husbands $(n=1213)$} \\
\hline No education & 354 & 302 & $656(54.1)$ \\
\hline Primary education & 246 & 133 & $379(31.2)$ \\
\hline Secondary and above & 150 & 28 & $178(14.7)$ \\
\hline \multicolumn{4}{|l|}{ Wealth status } \\
\hline Poor & 349 & 243 & $592(46.2)$ \\
\hline Middle & 157 & 113 & $270(21.1)$ \\
\hline Rich & 256 & 163 & $419(32.7)$ \\
\hline \multicolumn{4}{|c|}{ Birth order of the last child } \\
\hline 1 & 212 & 76 & $288(22.5)$ \\
\hline $2-4$ & 392 & 269 & $661(51.6)$ \\
\hline $5+$ & 158 & 174 & $332(25.9)$ \\
\hline \multicolumn{4}{|l|}{ Family size } \\
\hline $1-3$ & 212 & 98 & $310(24.2)$ \\
\hline $4-6$ & 406 & 263 & $669(52.2)$ \\
\hline$>=7$ & 144 & 158 & $302(23.6)$ \\
\hline \multicolumn{4}{|c|}{$\begin{array}{l}\text { Interval between the preceding non-first } \\
\text { births(n=993) }\end{array}$} \\
\hline$<24$ months & 23 & 23 & $46(4.6)$ \\
\hline 24-33 months & 123 & 122 & $245(24.7)$ \\
\hline 34-59 months & 358 & 272 & $630(63.4)$ \\
\hline$>=60$ months & 45 & 27 & $72(7.3)$ \\
\hline \multicolumn{4}{|c|}{ Intendedness of the last pregnancy } \\
\hline Intended & 667 & 395 & 1062 \\
\hline Unintended & 95 & 124 & 219 \\
\hline \multicolumn{4}{|c|}{ Menses resumed after last birth } \\
\hline Yes & 605 & 165 & $770(60.1)$ \\
\hline No & 157 & 354 & $511(39.9)$ \\
\hline
\end{tabular}




\begin{tabular}{llll}
\hline Yes & 390 & 121 & $511(39.9)$ \\
\hline No & 372 & 398 & $770(60.1)$ \\
\hline $\begin{array}{l}\text { Initiation time of first ANC after } \\
\text { conception (n=898) }\end{array}$ & & & \\
$\begin{array}{l}\text { First trimester } \\
\text { Second trimester }\end{array}$ & 121 & 13 & $134(14.9)$ \\
\hline $\begin{array}{l}\text { Third trimester } \\
\text { Place of delivery }\end{array}$ & 409 & 206 & $615(68.5)$ \\
\hline Healthcare facility & 74 & 75 & $149(16.6)$ \\
\hline Home & 482 & 127 & $609(47.5)$ \\
\hline $\begin{array}{l}\text { Mode of delivery(n=615) } \\
\text { SVD }\end{array}$ & 280 & 392 & $672(52.5)$ \\
\hline $\begin{array}{l}\text { Others(C/S, Assisted) } \\
\text { At least one PNC within 6weeks after }\end{array}$ & 392 & & $501(81.5)$ \\
birth & 94 & 129 & $114(18.5)$ \\
\hline Yes & 457 & 20 & \\
\hline No & 305 & 105 & $562(43.9)$ \\
\hline $\begin{array}{l}\text { Early PNC } \\
\text { (within 48-72 hours after birth) }\end{array}$ & & 419 & $719(56.1)$ \\
\hline Yes & 172 & & \\
\hline No & 590 & 20 & $192(15.0)$ \\
\hline
\end{tabular}

*SVD= Spontaneous Vaginal Delivery; $\mathrm{C} / \mathrm{S}=$ Caesarean Section; Assisted delivery includes vacuum and forceps deliveries

\section{Results from survival analysis}

Among all the respondents, 59.5\% (95\% CI: 56.8\%-62.2\%) had started using any modern contraceptive method after the last birth while the remaining were right-censored as of the time of the survey. Contraceptive users have contributed 11,737 months (978 women-years) of follow up, with an average follow-up time of 9.16 (95\% CI=8.96-9.37) months (Fig 1).

\section{Fig1. Kaplan Meier survival function curve showing time to modern contraceptive use} after birth among reproductive-age women in West Gojjam Zone, Northwest Ethiopia, and June 2018

Using the life-table method, we presented the probability of initiating contraception at 2, 6, 9, and 12 months after birth. By the second month after child birth, only 11.1 percent of the postpartum women surveyed started to use a contraceptive method. The proportion of users then increased steadily over the months reaching $25.9 \%, 37.7 \%$, and $59.5 \%$ at 6,9 , and 12 months, respectively (Table 2). 
Table 2. Survivor function of postpartum modern contraceptive use among postpartum women in West Gojjam Zone, Northwest Ethiopia, June, 2018

\begin{tabular}{lllllll}
\hline $\begin{array}{l}\text { Beginning } \\
\text { time } \\
\text { (months) }\end{array}$ & $\begin{array}{l}\text { Total number } \\
\text { of exposure }\end{array}$ & $\begin{array}{l}\text { Failure } \\
\text { (contraceptive } \\
\text { users) }\end{array}$ & Survivor function & SE & 95\% CI & \\
\hline 1 & 1281 & 4 & 0.9969 & 0.0016 & 0.9917 & 0.9988 \\
2 & 1277 & 138 & 0.8891 & 0.0088 & 0.8707 & 0.9051 \\
6 & 995 & 46 & 0.7408 & 0.0122 & 0.7159 & 0.7639 \\
9 & 844 & 46 & 0.6230 & 0.0135 & 0.5958 & 0.6489 \\
12 & 657 & 138 & 0.4052 & 0.0137 & 0.3782 & 0.4319 \\
\hline
\end{tabular}

The illustrations in figure 2 provide insights into the features of the differences in the Kaplan-

Meier survival curves by selected maternal characteristics. Clearly, the overall estimated survivor function showed that mothers started taking modern contraception after the $2^{\text {nd }}$ month of the last birth. It is also evident that the survival curves are substantially different among women whose first antenatal visit was during the first trimester, during the second trimester, or started in the third trimester. Similarly, the curves differ for various educational categories of women, the number of antenatal follow-ups, place of residence, and history of menstrual resumption after birth during the first12 months following the last childbirth (Fig 2a-f).

Fig 2: Kaplan Meier estimate curve of postpartum family planning use within 12 months from childbirth by selected characteristics of women, Northwest Ethiopia, June 2018

Before we fit the final model, the observed difference in survival experiences in different groups was also assessed using the log-rank test. The variables considered include maternal age, maternal and paternal educational attainment, frequency and timing of first ANC follow up, type of delivery, postnatal care within the first two days after birth, PHC to population ratio, family size, and household wealth index.

The log-rank test result revealed that each of the covariates, except for facility to population ratio, has a significant Wald test when using $\alpha=20 \%$. However, we kept facility to population 
ratio in the final model as access to the healthcare facility was considered an important variable in different literature. Moreover, the sample size is sufficient to accommodate more predictors. Then, all the covariates that were selected in the log- rank test at a $20 \%$ level of significance were fitted in the parametric shared frailty models of exponential, Weibull, log-logistic, and lognormal distributions by using cluster (kebele) as frailty term. The shared frailty model with the Weibull baseline hazard function had the smallest AIC value (Table 2) than the other frailty models and hence was selected to describe time-to-postpartum contraception data. The AIC values for all the parametric frailty models are summarized in table 3 below. 269 information criteria, June 2018.

\begin{tabular}{llllll}
\hline $\begin{array}{l}\text { Baseline } \\
\text { distribution }\end{array}$ & $\begin{array}{l}\text { Frailty } \\
\text { distribution }\end{array}$ & $\begin{array}{l}\text { Log- } \\
\text { likelihood }\end{array}$ & K & c & AIC Value \\
\hline Exponential & Gamma & -1403.4963 & 10 & 1 & 2828.9926 \\
Weibull & Gamma & -602.7483 & 10 & 2 & 1229.4960 \\
Log-logistic & Gamma & -1343.2516 & 10 & 2 & 2710.5032 \\
Log-normal & Gamma & -1333.658 & 10 & 2 & 2691.316 \\
\hline
\end{tabular}

$* \mathrm{AIC}=-2 \ln \mathrm{L}+2(\mathrm{k}+\mathrm{c})$, where $\mathrm{c}$ is the number of model-specific distributional parameters \& $k$ is the number of model covariates.

273 The effect of clustering (unobserved heterogeneity) between the clusters (Kebeles) was tested using the likelihood ratio test (LRT). The results of this test revealed that the variance of the

275 random term, $\theta$ (theta), was estimated to be 0.056 and it was statistically significant at the $5 \%$

276 level of significance. This indicated that the variance is significantly different from zero, and 277 unobservable factors cannot be ignored. Also, Kendall's tau $(\tau)$ value of 0.027 , suggests a 278 positive correlation between times to contraception within the clusters (Table 4). 
Predictors of time to contraceptive use after birth

\section{Goodness of fit of the final model}

280 The overall goodness of fit for the final model was checked by the Cox-Snell residuals plot. As

281 depicted in figure 3 below, the plot of the residuals of the fitted model is fairly closer to the $45^{0}$

282 straight line of the origin with slight variability in the right-hand tail, indicating that this model

283 had a better fit to the data. Note that some variability about the $45^{\circ}$ line is expected even with

284 well-fitted survival models, particularly in the right-hand tail, because of the reduced effective 285 sample caused by prior failures and censoring[25] (Figure3).

Figure 3. Cumulative hazard of Cox-Snell residuals

\section{Multivariable Survival Analysis result}

289 After controlling for other factors, the use of maternal health care services, and educational status

290 of the women were found to be the sole predictors of postpartum modern contraceptive methods 291 use.

292 The likelihood of initiating contraceptive use for women who had four or more antenatal visits 293 was 59 percent (aHR =1.59; 95\% CI: 1.22-2.06) higher than for women who had less number of 294 visits. Similarly, women who did start their first antenatal visit within the first trimester were 295 twice (aHR $=2.03 ; 95 \%$ CI: 1.28-3.21) more likely to initiate contraceptive use during the first 296 year postpartum than women who made the service in the third trimester. Women who received 297 postnatal care within the first three days after birth were also1.39 times more likely to use 298 modern contraceptive methods during the extended postpartum period than their counterparts $299 \quad(\mathrm{aHR}=1.39 ; 95 \%$ CI: $1.12-1.73)$.

300 Women's educational status was positively related to the early initiation of modern contraceptive 301 methods. The likelihood of initiating modern contraceptives in the extended postpartum was 
$30 \%$ higher among women who attained at least primary education compared with those who

had no formal education, $(\mathrm{aHR}=1.30 ; 95 \% \mathrm{CI}$ : $1.02-1.66)$.

However, in this multivariable analysis, no statistical difference was observed in contraceptive

uptake between women of different wealth status, area of residence, mode of delivery, and the

number of health facilities per total population (facility density) in the district.

Table 4. Results of Multivariable analysis of time-to-contraceptive after child birth by women aged 15-49 who had their most recent birth within 12 months preceding the survey, West Gojjam Zone, Ethiopia, June 2018

\begin{tabular}{|c|c|c|c|c|}
\hline \multicolumn{5}{|c|}{ Hazard Ratio (95\% CI) } \\
\hline Variables & $\begin{array}{l}\text { Unadjusted } \\
\text { HF }=\end{array}$ & $95 \% \mathrm{CI}$ & aHR & $95 \% \mathrm{CI}$ \\
\hline \multicolumn{5}{|l|}{ Residence } \\
\hline Urban & 1.77 & $1.46,2.15$ & 0.98 & $0.74,1.32$ \\
\hline Rural & 1.00 & --------- & 1.00 & $\ldots \ldots$ \\
\hline Age of women & 0.95 & $0.94,0.97$ & 0.99 & $0.97,1.02$ \\
\hline \multicolumn{5}{|c|}{ Educational status of women } \\
\hline No education & 1.00 & -------- & 1.00 & ----- \\
\hline Primary education & 1.95 & $1.66,2.30$ & 1.298 & $1.02,1.66^{* *}$ \\
\hline At least secondary & 3.37 & $2.75,4.12$ & & $0.98,1.84$ \\
\hline & & & 1.344 & \\
\hline Family size & 0.88 & $0.84,0.91$ & 0.99 & $0.91,1.09$ \\
\hline \multicolumn{5}{|l|}{ Wealth status } \\
\hline Poor & 1.00 & --- & 1.00 & --- \\
\hline Medium & 1.03 & $0.85,1.25$ & 0.96 & $0.73,1.25$ \\
\hline Rich & 0.99 & $0.84,1.18$ & 1.06 & $0.85,1.32$ \\
\hline \multicolumn{5}{|l|}{ Population per PHC } \\
\hline$<=25,000$ population & 1.01 & $0.71,1.44$ & 1.42 & $0.94,2.12$ \\
\hline$>25,000$ population & 1.00 & -------- & 1.00 & ---- \\
\hline \multicolumn{5}{|l|}{ Attended 4+ANC visits } \\
\hline Yes & 2.48 & $2.13,2.88$ & 1.59 & $1.22,2.06 * *$ \\
\hline No & 1.00 & ------ & 1.00 & ---- \\
\hline \multicolumn{5}{|l|}{ Timing of the first ANC } \\
\hline First trimester & 4.49 & $3.32,6.08$ & 2.03 & $1.28,3.21 * *$ \\
\hline Second trimester & 1.67 & $1.30,2.15$ & 1.43 & $0.97,2.12$ \\
\hline Third trimester & 1.00 & ------- & 1.00 & ------ \\
\hline \multicolumn{5}{|l|}{ Mode of delivery } \\
\hline SVD & 0.79 & $0.63,0.99$ & 0.81 & $0.62,1.04$ \\
\hline Other (c/s, instrumental) & 1.00 & ----- & 1.00 & --- \\
\hline \multicolumn{5}{|c|}{ Early PNC care (within 2-3 days after birth) } \\
\hline Yes & 3.31 & $2.78,3.96$ & 1.39 & $1.12,1.73 * *$ \\
\hline
\end{tabular}




\begin{tabular}{lccc}
\hline No & 1.00 & ---- & --- \\
$\boldsymbol{\theta}$ value (theta) & & 0.056 & 0.027 \\
$\boldsymbol{\tau}$ (tau Kendall) & -602.7483 \\
Log-likelihood(LL) & & \\
\hline
\end{tabular}
327 heterogeneity. $C / S=$ Caesarian Section

\section{Discussion}

** significant at 0.05level of significance, HR=Hazard Ratio; Ahr=Adjusted Hazard Ratio; CI=Confidence Interval;

$L R T=$ Likelihood ratio test of theta $(\theta)=0$ at chi-squares with 0 and 1 degrees of freedom; Kendall's tau $(\tau)=\theta / \theta+2$, where $\tau=\epsilon(0,1) .95 \%$ Confidence interval

This study has investigated the association of socioeconomic, demographic, and environmental factors with the likelihood of postpartum contraceptive use by accounting for gamma- distributed shared frailties at cluster-level.

In this study, we used kebele as a clustering (frailty) effect on modeling the determinants of time-to-contraception after birth. The statistically significant effect of the frailty terms between different clusters revealed that the observed covariates included in the analysis were not able to account for all the variability in women's survivorship. We postulate this variability to be the combined effect of various factors that cannot be easily measured or observed at community or health facility levels including culture and social norms[15]. The significant level of frailty terms in our study might have biased our results if we had not taken them into account [21]. Our interpretation, therefore, was based on the shared survival frailty model that accounts for the

In this study, modern contraceptive practice during the extended postpartum period was found to be $59.5 \%$ (95\% CI: $56.8-62.2 \%$ ). The finding is comparable with the finding of the study done in Debre Tabor town, Northwest Ethiopia 63.0\% (95\% CI: 59\%; 67.4\%) [26]. This result was, however, higher than the $31.7 \%$ prevalence in Southern Ethiopia[20], $45.4 \%$ in western 
332 Ethiopia[27], and 29.3\% in Northern Ethiopia[19], and lower when compared to the studies 333 done in Hosana town (72.9\%), Addis Ababa, Ethiopia (80.3\%), and 86.3\% of Kenya [28-30].

334 The low level of contraceptive use found in this study might reflect the over- reliance of lactating 335 women on breastfeeding and menstruation status. It is evidenced that these group of women 336 oftentimes do not realize that they are at risk of pregnancy when they are amenorrheic or 337 breastfeeding $[15,20,26]$. Though breastfeeding is universal in Ethiopia, and exclusive 338 breastfeeding up to 6 months after birth is an important contraceptive method which is highly 339 recommended by the Ministry of Health of Ethiopia, the status of exclusive breastfeeding in the 340 country is less than the global recommendations [31].

341 The main finding of this analysis is that women who started using modern contraceptive methods 342 during the extended postpartum period were characterized by high coverage (four or more visits) 343 and proper timing (first visit in first trimester) of antenatal care. Yet, the results of studies done 344 in Ethiopia and elsewhere $[10,18,28]$ showed that postpartum use of modern contraception was 345 not affected by antenatal care. The variation could be attributed to the difference in the study 346 design; whereas our study accounted for the hierarchical structure and tried to adjust for 347 individual and community characteristics, the other studies were done using flat models that 348 inherently assume the population to be homogeneous. It is evidenced that frailty models offer 349 unobserved heterogeneity into models for survival data as random effects [21].

350 Nonetheless, the observed association between prenatal care and contraceptive use is not unique 351 to this study and has been reported in earlier studies from Ethiopia [20,27,32], and other 352 countries $[8,16]$. These studies showed a dose-response type of relationship between antenatal 353 care and postpartum contraception adoption; that is the likelihood of using postpartum 354 contraception increased when women had frequent antenatal contacts. Our result also 
355 demonstrated a significant association between postpartum contraceptive use and early postnatal 356 care, which is in agreement with other studies [20,27].

357 The positive effects of maternity services on contraceptive uptake might be explained due to the 358 effect of the counseling sessions and promotional efforts made during each visit. It has been 359 indicated that each maternity services improve clients' relationships with health workers and their familiarity with the health care systems [33]. Besides, counseling and information can help women avoid social barriers and, in turn, encourage them to use health services in the future

362 [34]. Therefore, cognizant of the fact that only a few Ethiopian women have gotten antenatal and early postnatal services [3], there is a strong need to promote programs that target women who 364 do not get these services as a strategy to promote postpartum modern contraceptive use.

365 There are inconsistent pieces of evidence in the correlation between women's education and postpartum contraception adoption. A study done in Northwest Ethiopia, for example, did not 367 show any association between female education and postpartum contraceptive uptake[18]. On 368 the other hand, in line with the previous researches[10,15], the result of our study also confirms 369 that educated women have a higher hazard of contraceptive use when compared to mothers with no formal education. Women's education could impact modern contraceptive uptake in different 371 mechanisms: improving access to contraceptive alternatives, and helping them in understanding 372 the health benefits of the available contraceptive commodities [35] might be among the possible 373 reasons. Education might also improve the bargaining power of women to negotiate sex, and 374 their ability to make their own decisions, including fertilities desires [36].

375 Results from various studies have found conflict of evidence on the link between household 376 wealth status and the use of postpartum contraception; in some settings, it appears to be 377 associated with contraceptive use; Hounton and colleagues, for example, reported financial 378 constraint as a barrier to adopt postpartum contraception[10]. However, no statistically 
379 significant difference was observed in contraceptive uptake between women of different wealth 380 status in our study, which is in line with the results of some other studies[15,28]. This lack of 381 variation in contraceptive use by wealth status in our study might be attributed to the 382 introduction of healthcare financing reforms by the government of Ethiopia, which includes 383 social and community based health insurance schemes, and charge free maternity services in 384 public health facilities, among others[37]. Concerning this, Dzakpasu et al reported that poor 385 women were unwilling to use the formal health sector if they must pay for maternal health 386 services[38]. The Health Extension Program in Ethiopia, which brought family planning services 387 to the community where they live, might be another reason for the lack of variation between rich 388 and poor women. Health extension workers are deployed in pairs, two for every kebele, and 389 affiliated with each kebele's health post to provide key health services at a community level, 390 including family planning services since launched in 2003 [39].

392 Despite we tried to estimate unbiased parameter estimates after accounting for the frailty effect, 393 the study results should also be interpreted in light of certain limitations. The reliability of this 394 study depends on the mother's recall of past events regarding the processes of maternal health 395 care and therefore may be subject to recall bias. In addition, the study focused merely on the 396 health coverage of maternal services as main predictors, yet coverage alone might not be a 397 warranty for postpartum contraceptive use if quality was insufficient[40].

\section{Conclusions}

399 In conclusion, the use of postpartum modern contraception was low despite the provision of 400 charge-free services in all public health facilities. Postpartum modern contraception use was 401 associated with increased coverage of the key maternal services, particularly the antenatal and 
402 postnatal cares . The observed strong effect of antenatal and early postnatal services strengthens

403 the argument that integrating the key maternity could enhance the use of postpartum modern 404 contraception. Moreover, the significant level of variance of unobserved community effect also 405 underscores the importance of disaggregated data for evidence-based policymaking and program 406 designing in the study area in particular and the country in general.

\section{Acknowledgments}

408 We would like to express our deepest gratitude to the Amhara regional health bureau, West

409 Gojjam Zone Health Department, and district health offices. We also extend our

410 acknowledgment to the data collectors, supervisors and study participants.

\section{Author contributions}

412 Conceptualization: Amanu Aragaw Emiru

413 Data curation: Amanu Aragaw Emiru, Getu Degu Alene, Gurmesa Tura Debelew

414 Formal analysis: Amanu Aragaw Emiru, Getu Degu Alene, Gurmesa Tura Debelew

415 Methodology: Amanu Aragaw Emiru, Getu Degu Alene, Gurmesa Tura Debelew

416 Supervision: Getu Degu Alene, Gurmesa Tura Debelew

417 Writing - original draft: Amanu Aragaw Emiru

418 Writing -review \& editing: Amanu Aragaw Emiru, Getu Degu Alene, Gurmesa Tura Debelew 419 REFERENCES

1. Alkema L, Chou D, Hogan D, Zhang S, Moller A-B, et al. (2016) Global, regional, and national levels and trends in maternal mortality between 1990 and 2015, with scenario-based projections to 2030: a systematic analysis by the UN Maternal Mortality Estimation Inter-Agency Group. The Lancet 387: $462-474$. 
Predictors of time to contraceptive use after birth

424

425

426

427

428

429

430

431

432

433

434

435

436

437

438

439

440

441

442

443

444

445

446

447

448

449

450

451

452

453

454

455

456

457

458

2. WHO, Unicef (2014) Trends in maternal mortality: 1990 to 2013: estimates by WHO, UNICEF, UNFPA, The World Bank and the United Nations Population Division.

3. Central Statistical Agency (CSA) [Ethiopia] and ICF. 2016 Ethiopia Demographic and Health Survey Key Findings. Addis Ababa, Ethiopia, and Rockville, Maryland, USA. CSA and ICF, 2017.

4. Berhan Y, Berhan A (2014) Review of maternal mortality in Ethiopia: a story of the past 30 years. Ethiopian journal of health sciences 24: 3-14.

5. Kerber KJ, de Graft-Johnson JE, Bhutta ZA, Okong P, Starrs A, et al. (2007) Continuum of care for maternal, newborn, and child health: from slogan to service delivery. The Lancet 370: 13581369.

6. World Health Organization (2013) Programming strategies for postpartum family planning. Geneva: WHO; 2013.

7. Tran NT, Gaffield ME, Seuc A, Landoulsi S, Yamaego WME, et al. (2018) Effectiveness of a package of postpartum family planning interventions on the uptake of contraceptive methods until twelve months postpartum in Burkina Faso and the Democratic Republic of Congo: the YAM DAABO study protocol. BMC health services research 18: 439.

8. Achyut P, Mishra A, Montana L, Sengupta R, Calhoun LM, et al. (2016) Integration of family planning with maternal health services: an opportunity to increase postpartum modern contraceptive use in urban Uttar Pradesh, India. Journal of Family Planning and Reproductive Health Care 42: 107115.

9. Lawn JE, Kinney MV, Belizan JM, Mason EM, McDougall L, et al. (2013) Born too soon: accelerating actions for prevention and care of 15 million newborns born too soon. Reproductive health 10 : S6.

10. Hounton S, Winfrey W, Barros AJ, Askew I (2015) Patterns and trends of postpartum family planning in Ethiopia, Malawi, and Nigeria: evidence of missed opportunities for integration. Global health action 8: 29738.

11. Moore Z, Pfitzer A, Gubin R, Charurat E, Elliott L, et al. (2015) Missed opportunities for family planning: an analysis of pregnancy risk and contraceptive method use among postpartum women in 21 low-and middle-income countries. Contraception 92: 31-39.

12. Tadele A, Abebaw D, Ali R (2019) Predictors of unmet need for family planning among all women of reproductive age in Ethiopia. Contraception and reproductive medicine 4: 6.

13. Tegegn M, Arefaynie M, Tiruye TY (2017) Unmet need for modern contraceptives and associated factors among women in the extended postpartum period in Dessie town, Ethiopia. Contraception and reproductive medicine 2: 21.

14. Sonalkar S, Gaffield ME (2017) Introducing the World Health Organization postpartum family planning compendium. International Journal of Gynecology \& Obstetrics 136: 2-5. 
Predictors of time to contraceptive use after birth

15. RamaRao S, Ishaku S, Liambila W, Mane B (2015) Enhancing contraceptive choice for postpartum women in sub-Saharan Africa with the progesterone vaginal ring: a review of the evidence. Open Access Journal of Contraception 6: 117.

16. Pasha O, Goudar SS, Patel A, Garces A, Esamai F, et al. (2015) Postpartum contraceptive use and unmet need for family planning in five low-income countries. Reproductive health 12: S11.

17. Langlois ÉV, Miszkurka M, Zunzunegui MV, Ghaffar A, Ziegler D, et al. (2015) Inequities in postnatal care in low-and middle-income countries: a systematic review and meta-analysis. Bulletin of the World Health Organization 93: 259-270G.

18. Tessema GA, Mekonnen TT, Mengesha ZB, Tumlinson K (2018) Association between skilled maternal healthcare and postpartum contraceptive use in Ethiopia. BMC pregnancy and childbirth 18: 172.

19. Embafrash G, Mekonnen W (2019) Level and Correlates of Unmet Need of Contraception among Women in Extended Postpartum in Northern Ethiopia. International journal of reproductive medicine 2019.

20. Dona A, Abera M, Alemu T, Hawaria D (2018) Timely initiation of postpartum contraceptive utilization and associated factors among women of child bearing age in Aroressa District, Southern Ethiopia: a community based cross-sectional study. BMC public health 18: 1100.

21. Balan TA, Putter H (2019) Nonproportional hazards and unobserved heterogeneity in clustered survival data: When can we tell the difference? Statistics in medicine.

22. West Gojjam ZoneHealth Office. The 2016/17 Fiscal Year Annual performance report of West Gojjam Zone, Finote Selam ; July, 2017.

23. Central Statistical Agency (CSA) [Ethiopia] and ICF. Ethiopia Demographic and Health Survey 2016: Key Indicators Report . Addis Ababa E, and Rockville, Maryland, USA. CSA and ICF, 2016

24. Gutierrez RG (2002) Parametric frailty and shared frailty survival models. The Stata Journal 2: 22-44.

25. Cleves M, Gould W, Gould WW, Gutierrez R, Marchenko Y (2008) An introduction to survival analysis using Stata: Stata press.

26. Taye EB, Mekonen DG, Debele TZ (2019) Prevalence of post partum modern family planning utilization and associated factors among postpartum mothers in Debre Tabor town, North West Ethiopia, 2018. BMC research notes 12: 430.

27. Teka TT, Feyissa TR, Melka AS, Bobo FT (2018) Role of antenatal and postnatal care in contraceptive use during postpartum period in western Ethiopia: a cross sectional study. BMC research notes 11: 581.

28. Gejo NG, Anshebo AA, Dinsa LH (2019) Postpartum modern contraceptive use and associated factors in Hossana town. PloS one 14: e0217167. 
Predictors of time to contraceptive use after birth

29. Gebremedhin AY, Kebede Y, Gelagay AA, Habitu YA (2018) Family planning use and its associated factors among women in the extended postpartum period in Addis Ababa, Ethiopia. Contraception and reproductive medicine 3: 1.

30. Jalang'o R, Thuita F, Barasa SO, Njoroge P (2017) Determinants of contraceptive use among postpartum women in a county hospital in rural KENYA. BMC public health 17: 604.

31. Alebel A, Tesma C, Temesgen B, Ferede A, Kibret GD (2018) Exclusive breastfeeding practice in Ethiopia and its association with antenatal care and institutional delivery: a systematic review and meta-analysis. International breastfeeding journal 13: 31.

32. Tafere TE, Afework MF, Yalew AW (2018) Counseling on family planning during ANC service increases the likelihood of postpartum family planning use in Bahir Dar City Administration, Northwest Ethiopia: a prospective follow up study. Contraception and reproductive medicine 3: 28.

33. Tappis H, Kazi A, Hameed W, Dahar Z, Ali A, et al. (2015) The role of quality health services and discussion about birth spacing in postpartum contraceptive use in Sindh, Pakistan: a multilevel analysis. PloS one 10: e0139628.

34. Kongnyuy E, Mlava G, Broek N (2009) Facility-Based Maternal Death Review In Three Districts In The Central Region of Malawi. Women's health issues : official publication of the Jacobs Institute of Women's Health 19: 14-20.

35. Mekonnen W, Worku A (2011) Determinants of low family planning use and high unmet need in Butajira District, South Central Ethiopia. Reproductive health 8: 37.

36. Weitzman A (2017) The effects of women's education on maternal health: Evidence from Peru. Social Science \& Medicine 180: 1-9.

37. Zelelew H (2018) Health care financing reform in Ethiopia: improving quality and equity. Bethesda (MD): Health Systems 20: 20.

38. Dzakpasu S, Powell-Jackson T, Campbell O (2014) Impact of user fees on maternal health service utilization and related health outcomes: a systematic review. Health policy and planning 29: 137.

39. Wang H, Tesfaye R, NV Ramana G, Chekagn CT (2016) Ethiopia health extension program: an institutionalized community approach for universal health coverage: The World Bank.

40. WHO (2016) WHO recommendations on antenatal care for a positive pregnancy experience. 


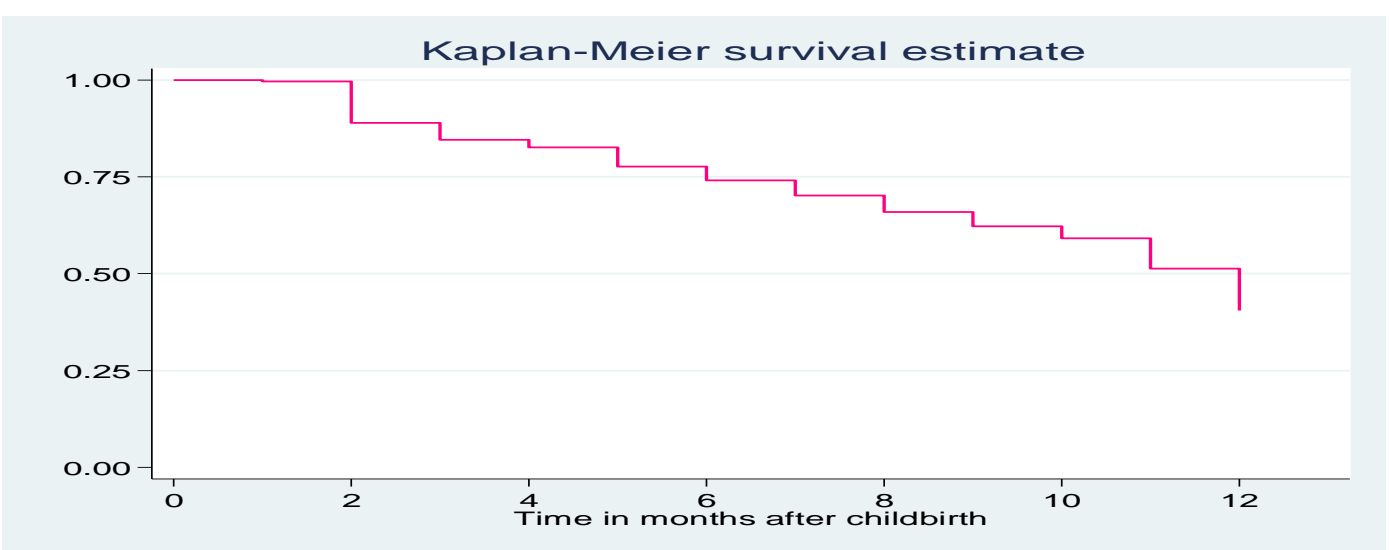

Fig 1
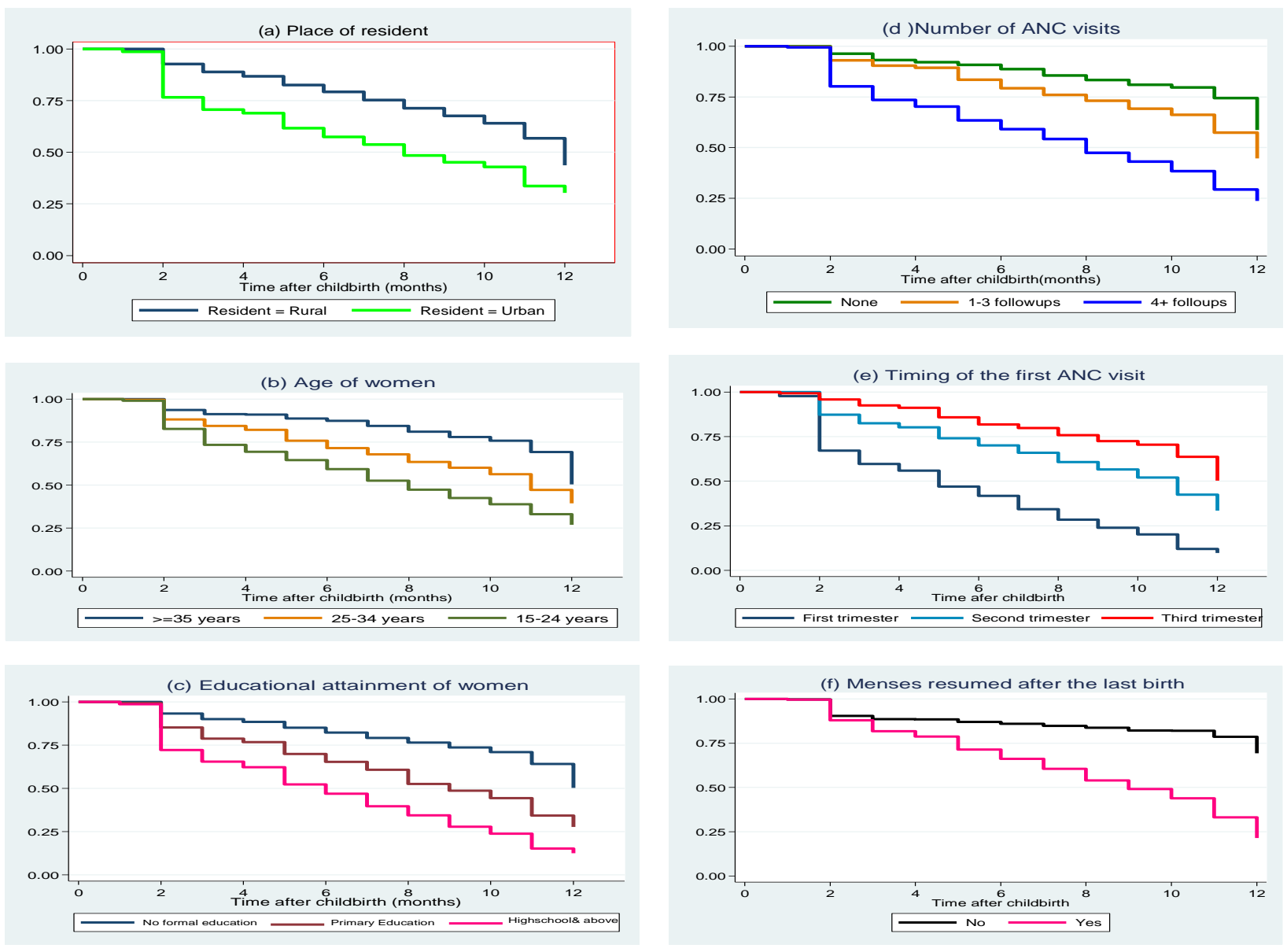

Fig 2 


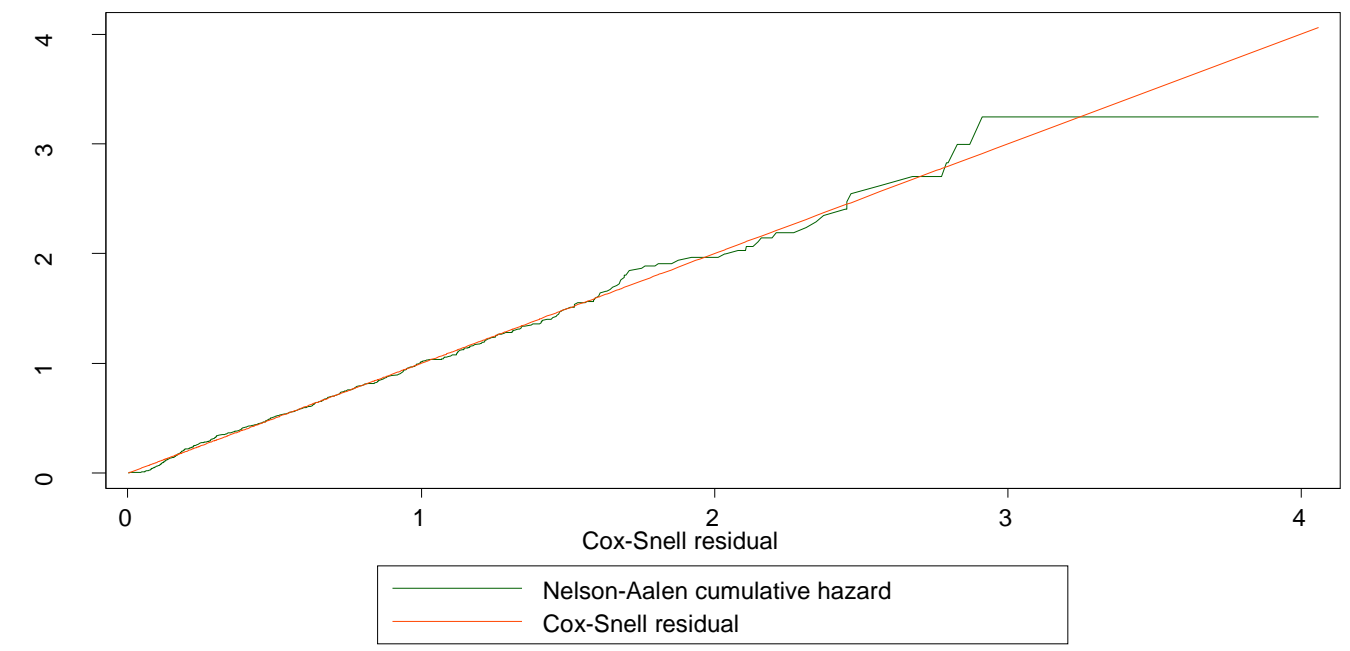

Fig 3 